\title{
Electrostatic Potential Fluctuations on Oxide-Passivated Si(111) Surfaces Measured Using Integrated Scanning Probe Microscopy*
}

\author{
Leonid Bolotov, ${ }^{\dagger}$ Tetsuya Tada, Masanori Iitake, Masayasu Nishizawa, and Toshihiko Kanayama \\ Nanodevice Innovation Research Center, National Institute of Advanced Industrial Science and Technology, \\ Higashi 1-1-1, Tsukuba, Ibaraki 305-8562, Japan
}

(Received 25 October 2010; Accepted 2 February 2011; Published 26 March 2011)

\begin{abstract}
Variations of the electrostatic potential were investigated for oxide-passivated $n$-Si(111) surfaces with atomically flat terraces by measuring the force acting on an ultra-sharp tungsten probe that was attached to the quartz resonator of an atomic force microscope. When the probe-sample gap maintained a constant tunneling current, an enhancement of electrostatic force with a lateral extent of $\sim 5 \mathrm{~nm}$ was observed around underlying donor atoms and charged defects. Additional variations of the surface potential and the probe-sample capacitance across the surface steps were associated with excess electric charge at the step edge. [DOI: 10.1380/ejssnt.2011.117]
\end{abstract}

Keywords: Scanning Tunneling Microscopy; Surface electronic phenomena; Sb; Silicon; Semiconductor-insulator interfaces

\section{INTRODUCTION}

Electrostatic potential fluctuations caused by interface charges and impurity atoms in the channel region of a modern electronic device can lead to variability in device performance [1]. On the other hand, a discrete dopant distribution enables attractive applications such as quantum computing [2] and single-electron devices [3]. Therefore, monitoring the charge/dopant atom distribution is a necessary step toward understanding the correlation between the location of dopant atoms and carrier transport in nanoscale electronic devices.

For characterization of subsurface electrical junctions, passivation of Si surfaces by hydrogenation or oxidation has been employed in order to reproducibly prepare uniform surfaces with a very low density of states in the $\mathrm{Si}$ band gap. High-resolution measurements of individual impurity charges have been demonstrated on passivated Si surfaces by scanning tunneling microscopy (STM) [48]. Local potential fluctuations induced by randomly distributed dopant atoms have been reported for Si $p-n$ junctions [9-11]. However, STM is limited to conductive surfaces, and is therefore inapplicable to the imaging of novel device structures, including insulator surfaces such as silicon-on-insulator (SOI) devices.

Alternatively, scanning Kelvin force microscopy (SKFM) seems a suitable technique for surface potential measurements at an atomic scale [12-17]. However, the sensitivity of SKFM to the surface electrical potential depends strongly on the probe geometry and the probesample distance. At large probe-sample distances, the measured potential is a weighted average over a wide surface area, resulting in degradation of the lateral resolution and sensitivity [18-20]. At short distances, the prevailing repulsive force between the terminal atoms of the probe tip and the surface makes the measurements insensitive to the electric potential of sub-surface charges $[12,13,17]$.

\footnotetext{
*This paper was presented at the 13th International Conference on Non-Contact Atomic Force Microscopy (NC-AFM2010), Ishikawa Ongakudo, Kanazawa, Japan, 31 July - 4 August, 2010.

${ }^{\dagger}$ Corresponding author: lbolotov@bk.tsukuba.ac.jp; Present address: Institute of Applied Physics, University of Tsukuba, Tennodai 1-1-1, Tsukuba 305-8573, Japan
}

In this work, we investigated oxide-passivated $\mathrm{Si}(111)$ surfaces with atomically flat terraces using STM combined with non-contact atomic force microscopy (ncAFM) to map the local dopant concentration. With a small probe vibration amplitude of $300 \mathrm{pm}$, we demonstrated the ability to detect variation of probe-sample interactions caused by subsurface charges of impurity atoms and electric charge near surface step edges, when the probe-sample gap was maintained with a constant tunneling current.

\section{EXPERIMENTAL}

Figure 1(a) shows the experimental setup for the simultaneous measurement of tunnelling current $\left(I_{\text {tun }}\right)$ and force between the metal probe tip and the Si surface. We used an STM probe fabricated from a tungsten wire attached to a quartz force sensor and sharpened by an FIB technique in a manner reported in Ref. 21.

Figure 1(b) shows energy diagrams of a tunnel junction between a metal STM probe and an oxidized $n$-Si surface under a bias voltage causing carrier depletion at the surface. For passivated Si surfaces, the applied voltage $V_{\mathrm{S}}$ is shared between the vacuum gap ( $\left.V_{\text {gap }}\right)$ and the band-bending region $\left(V_{\mathrm{bb}}\right)$ beneath the surface [22]. Under the applied voltage $V_{\mathrm{S}}$, the electrostatic force gradient between the probe tip and the sample is expressed as [13]:

$$
\Delta f \propto \frac{\partial F}{\partial z}=-\frac{1}{2}\left(V_{\mathrm{S}}-C P D\right)^{2} \cdot \frac{\partial^{2} C}{\partial z^{2}},
$$

where $C$ is the effective tip-sample capacitance and $Z$ is the tip-sample gap. $C P D$, the contact potential difference, refers to the difference between the work function of the metal probe $\left(\phi_{\mathrm{M}}\right)$ and the Fermi energy of the underlying $\mathrm{Si}\left(E_{\mathrm{F}}\right)$, and is given as

$$
C P D=\frac{1}{q}\left(E_{\mathrm{F}}-\phi_{\mathrm{M}}\right),
$$

where $q$ is the elementary charge.

In our measurements, the force gradient was detected as a shift of the resonance frequency $(\Delta f)$ of a quartz oscillator when a sharp metal probe vibrated normal to the surface with an amplitude $(\Delta Z)$ of $300 \mathrm{pm}$ at a resonance frequency of $\sim 1 \mathrm{MHz}$. The force gradient map 

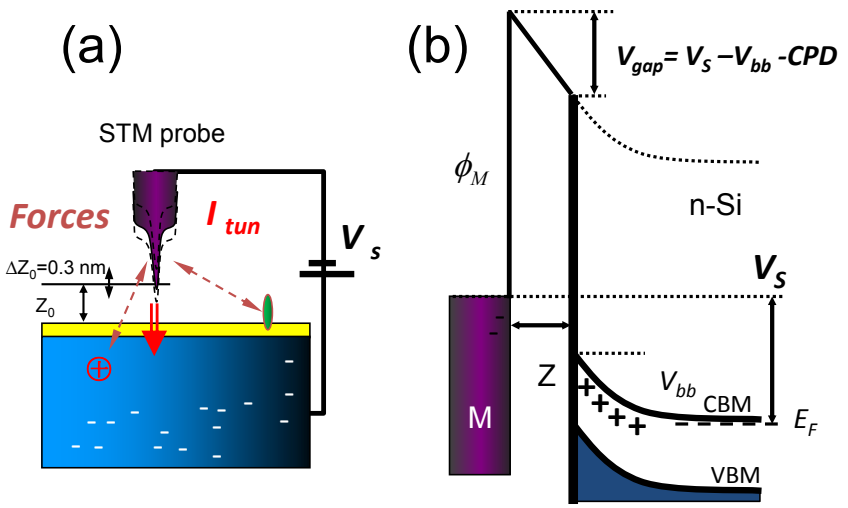

(c)

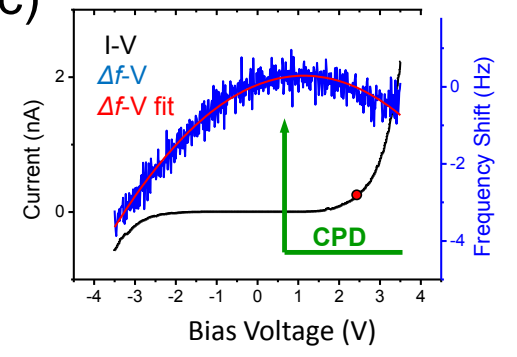

FIG. 1: (a) Experimental setup for simultaneous measurements of the tunnelling current $\left(I_{\text {tun }}\right)$ and electrostatic force between the metal probe tip and the oxidized $n$-Si surface. The mean gap $\left(Z_{o}\right)$ was maintained with a tunnelling current of $50 \mathrm{pA}$ and a bias voltage of $2.5 \mathrm{~V}$, while the probe vibrated with an amplitude $(\Delta Z)$ of $300 \mathrm{pm}$. (b) Energy band structure of a tunnel junction under bias voltage $V_{\mathrm{s}}$. CPD corresponds to the voltage at which the conduction $(\mathrm{CBM})$ and valence (VBM) bands in Si are flat. (c) Experimental $\left(I_{\text {tun }}-V_{\mathrm{s}}\right)$ and $\left(\Delta f-V_{\mathrm{s}}\right)$ spectra acquired simultaneously on the $\mathrm{Si}(111)$ surface while maintaining the mean vacuum gap.

(frequency shift map) was measured while the mean gap $(Z)$ maintained a tunnelling current of $50 \mathrm{pA}$ under a bias voltage of $2.5 \mathrm{~V}$. Typically, the measurement conditions corresponded to a gap of approximately $1 \mathrm{~nm}$, as estimated from the distance dependence of the tunnelling current [23]. When we moved the probe 300 pm closer to the surface, the tunneling current became unstable and the resonance frequency shift increased sharply to about $30 \mathrm{~Hz}$. This indicates that the minimum distance to the surface $\left(Z_{\min }\right)$ was larger than $600 \mathrm{pm}$. Therefore, the frequency shift gives a good approximation of the force gradient for $\Delta Z<Z_{\min }[24]$.

The CPD voltage and pre-factor, the second derivative of the effective probe-sample capacitance, were obtained from a fit of the $(\Delta f-V)$ spectrum to Eq. (1). Figure 1(c) shows typical $I-V$ and $(\Delta f-V)$ spectra acquired on oxidepassivated $\mathrm{Si}(111)$ surface by sweeping the bias voltage while holding the probe-sample gap set with a sample bias voltage of $3.0 \mathrm{~V}$ and a tunneling current of $50 \mathrm{pA}$. The fact that the fit agrees well with the experimental $(\Delta f-V)$ spectrum confirms the prevailing contribution of electrostatic force under our measurement conditions, and justifies the applicability of Eq. (1).

Furthermore, as the screening of charged donor atoms was reduced by carrier depletion at the surface, the detection of the attractive force between the probe tip and individual donor charges becomes possible. Therefore, this

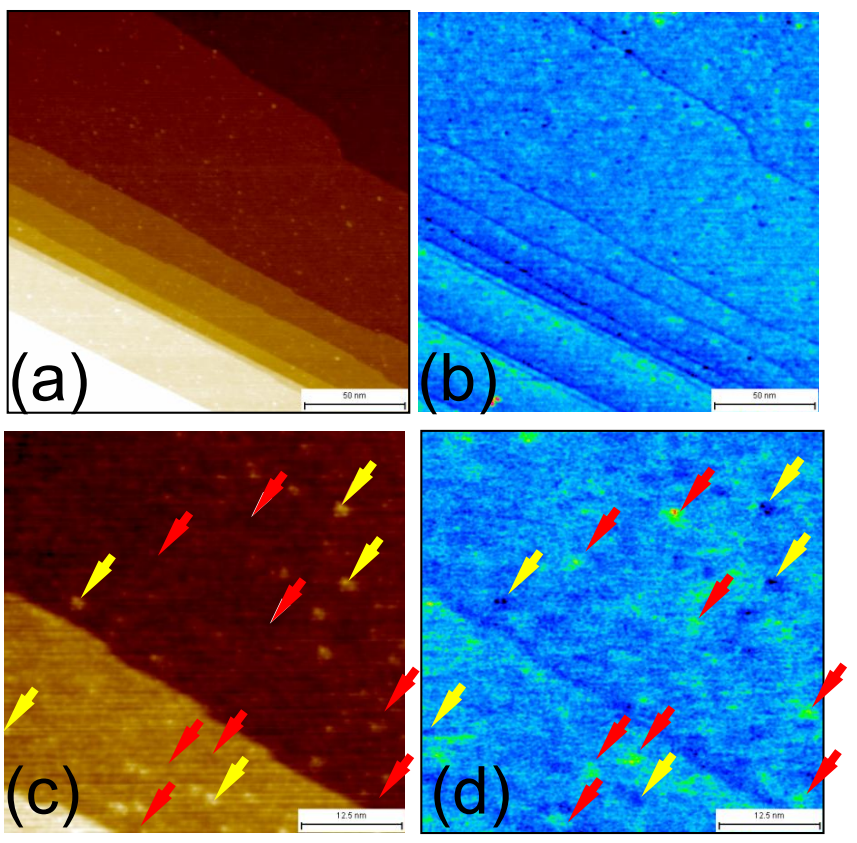

FIG. 2: STM topographs (a, c) and corresponding force gradient maps (b, d) of an oxidized $n$-Si(111) surface taken simultaneously at a bias voltage of $2.5 \mathrm{~V}$ and a current of $50 \mathrm{pA}$. Arrows in (c) and (d) indicate the positions of force fluctuation caused by sub-surface charges (red) and surface steps (yellow). The scale bar is $50 \mathrm{~nm}$ in (a, b) and $12 \mathrm{~nm}$ in (c, d). The color scale is $700 \mathrm{pm}$ in (a), $400 \mathrm{pm}$ in (c), and $7 \mathrm{~Hz}$ in (b, d).

is a sensitive method of monitoring variations in electrostatic forces between the STM probe and subsurface carriers, impurity charges, and surface states.

To examine the sensitivity and spatial resolution of the measurement technique, surfaces of $\mathrm{Si}(111)$ ( $\mathrm{Sb}$, $3 \times 10^{18} \mathrm{~cm}^{-3}$ ) with atomically flat terraces were prepared by flash annealing in an ultra-high vacuum (UHV) chamber. To eliminate surface dangling bonds, an ultra-thin oxide layer $0.3 \mathrm{~nm}$ thick was grown under an oxygen pressure of $2 \times 10^{-3} \mathrm{~Pa}$ at $600^{\circ} \mathrm{C}$. Additionally, the oxidized samples were annealed in a mixture of $\mathrm{H}_{2}: \mathrm{N}_{2}$ gases under a gas flow rate of $8 \mathrm{sccm}$ at $470^{\circ} \mathrm{C}$ for $30 \mathrm{~min}$. This preparation left an unpinned surface with a low density of states in the band gap of $\mathrm{Si}$, which is suitable for tunneling microscopy $[25,26]$.

\section{RESULTS AND DISCUSSION}

Figure 2 shows a set of STM images and corresponding force gradient maps for an oxide-passivated $\mathrm{Si}(111)$ surface. The sample surface had atomically flat terraces $20-80 \mathrm{~nm}$ wide, separated by steps $\sim 250 \mathrm{pm}$ in height running from the top-left to the bottom-right corner of the topographic image. A number of small protrusions $\sim 300 \mathrm{pm}$ in height can be seen in Fig. 2(c). The corresponding force gradient maps revealed differences in the interaction force acting on the STM probe across the step edges and on the flat terraces. Across the step edges, the frequency shift changed 3 -fold, from a mean value of $-1.1 \mathrm{~Hz}$ on the upper terrace to about $-3 \mathrm{~Hz}$ on the 

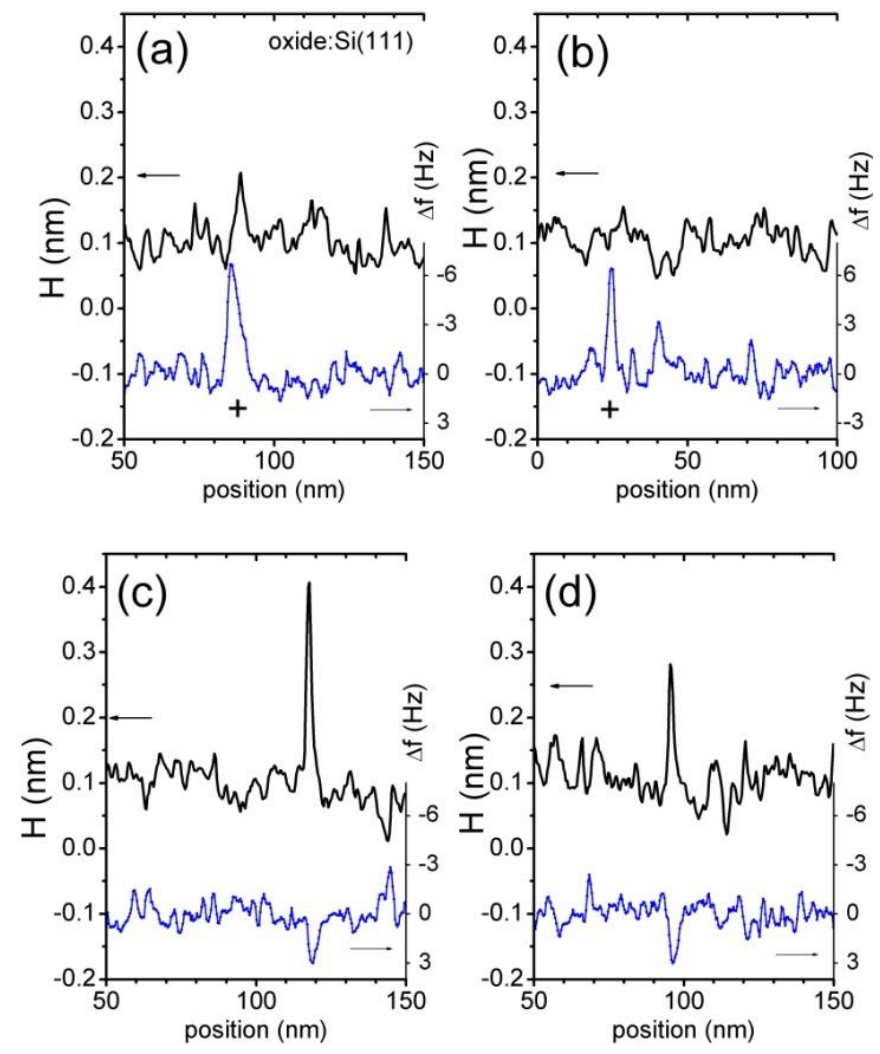

FIG. 3: Line profiles of height ( $H$, black line) and the frequency shift $(\Delta f$, blue line) on an atomically flat, oxidized $\mathrm{Si}(111)$ terrace in Fig. 2 across features of type II (a, b) and type I (c, d).

lower terrace. The enhanced interaction extended away from the step edge on the low terrace, suggesting it had its origin in long-range electrostatic forces. For narrow terraces, the effect was multiplied, as shown in the bottomleft corner of the image in Fig. 2(b). Note that the observed structure remained while the images were recorded during probe movement across the surface steps in either forward or backward scanning directions and with slower scanning speeds. Therefore, the observed structure was not a scanning artifact.

Two types of features were observed in the force gradient maps on flat terraces, as shown in Figs. 2(b, d). Type I features showed a decrease in the $\Delta f$ value from $-1.1 \mathrm{~Hz}$ to about $-3 \mathrm{~Hz}$, and coincided with small topographic protrusions, as indicated by yellow arrows in Figs. 2(c) and (d). For type I features, the correlation between the topographic protrusion $\sim 0.3 \mathrm{~nm}$ in height and the decrease in the frequency shift is evident in Figs. 3(c) and $(\mathrm{d})$.

Another type of features, marked by red arrows in Fig. 2, showed an increase in the $\Delta f$ value from $-1.1 \mathrm{~Hz}$ to $+6 \mathrm{~Hz}$, and were observed only in the force gradient map. The type II features showed a lateral extent of 5-10 nm, and varied in number from 6 to 9 in different $50 \times 50 \mathrm{~nm}^{2}$ areas, corresponding to an area density of $2-4 \times 10^{11} \mathrm{~cm}^{2}$.

The line profiles in Fig. 3 show the detailed differences between surface features on the oxidized $\mathrm{Si}(111)$ surface. The type II features showed an increase in frequency shift without any distinct topographic structure, as seen in

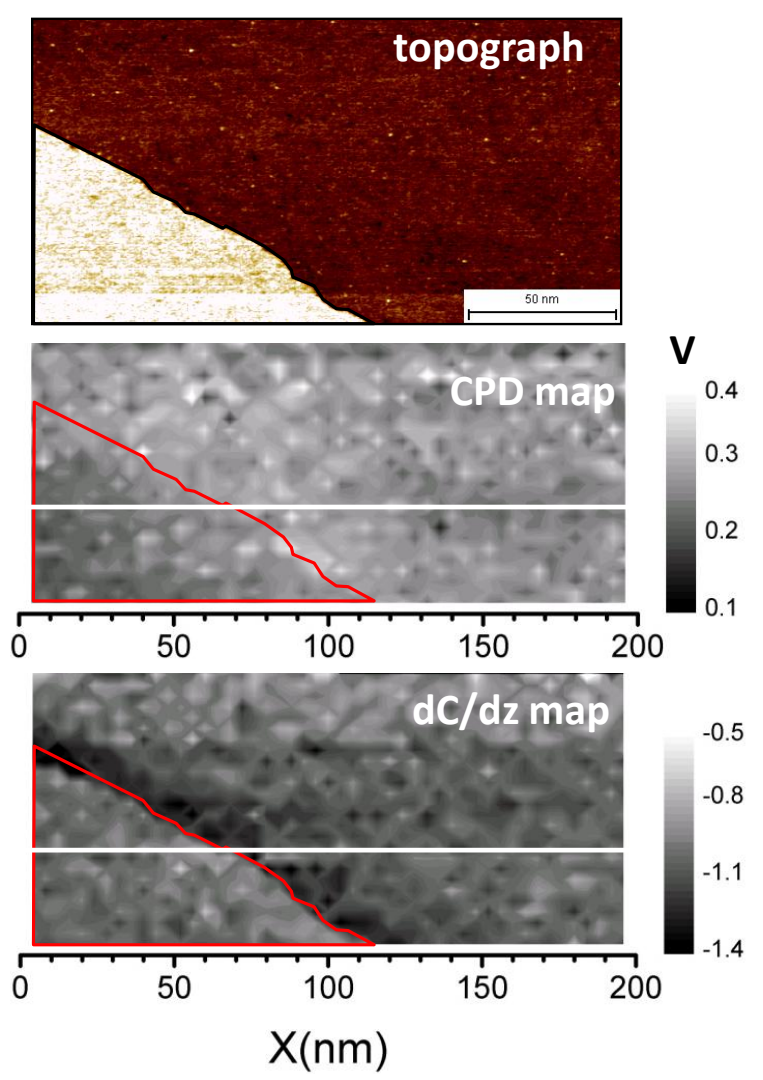

FIG. 4: STM topograph (top), and corresponding maps of CPD voltage (middle) and the derivative of probe-sample capacitance (bottom) obtained from fitting $\left(\Delta f-V_{\mathrm{s}}\right)$ spectra measured with a separation of $2 \mathrm{~nm}$. The atomic step edge on an oxidized $n$-Si(111) surface is indicated by the red line.

Figs. 3(a) and (b). A frequency shift of about $7 \mathrm{~Hz}$ was observed, which was larger than the noise level of $\pm 1 \mathrm{~Hz}$ obtained for the oxidized surface with a surface roughness of $\pm 50 \mathrm{pm}$. The absence of correlation with topographic structure suggests that the type II features originated in long-distance electrostatic interactions such as between the probe and a positive donor charge beneath the surface.

Interestingly, the density of type II features agrees with the expected number of donors in a $50 \times 50 \mathrm{~nm}^{2}$ area for a donor concentration of $3 \times 10^{18} \mathrm{~cm}^{-3}$ and a detection depth of $1 \mathrm{~nm}$. Because the screening by free carriers is reduced under a positive sample bias, the STM probe experiences the attractive force due to the electric charge of an individual dopant atom underneath the surface located closest to the probe. Here, a lateral extent of 5-10 nm was observed for individual features in the force gradient maps shown in Fig. 2. This value is comparable to the Bohr radius of a positive donor in $\mathrm{Si}$, and the lateral extent of $\sim 15 \mathrm{~nm}$ observed for phosphor and boron atoms in $\mathrm{Si}$ reported in the literature $[6,7]$.

To elucidate the origin of the fluctuations in the force gradient map shown in Fig. 2, a CPD map along an atomic terrace on the passivated $n$-Si(111) surface was obtained by measuring an array of $(\Delta f-V)$ spectra with a lateral separation of $2 \mathrm{~nm}$. The CPD map in Fig. 4 and the line profile in Fig. 5 showed a smooth variation of the surface potential across a $\sim 300$-pm-high surface step. We 


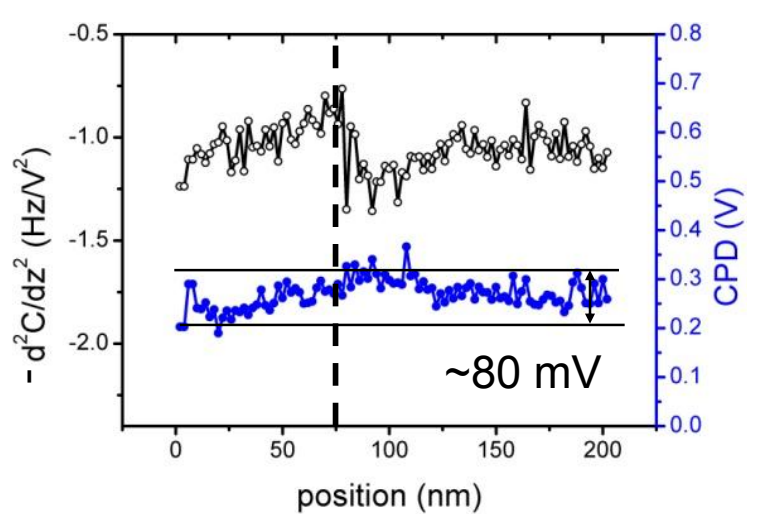

FIG. 5: Line profiles of CPD voltage (closed circles) and the derivative of the effective junction capacitance (open circles) across an atomic step along the white line in Fig. 4. The vertical broken line indicates the position of the step edge.

obtained a mean CPD voltage of $270 \mathrm{mV}$ and a standard deviation of $30 \mathrm{mV}$ at room temperature. The measured mean CPD voltage agrees with the Fermi energy position calculated for $\phi_{\mathrm{M}}=4.5 \mathrm{eV}$ and a doping concentration of $\sim 3 \times 10^{18} \mathrm{~cm}^{-3}$ determined by four-probe measurements.

Changes in the CPD voltage across the step correlate with changes in the derivative of the probe-sample capacitance (Fig. 4). While a variation of $\sim 80 \mathrm{mV}$ in the CPD voltage was measured across the atomic step, the second derivative of the capacitance (the pre-factor in Eq. (1)) changed 2-fold across the step edge. The capacitance variation extended $\sim 20 \mathrm{~nm}$ from the step edge, as can be seen in Fig. 4. We note that the surface potential is determined by the area density of electric charge, as is the capacitance. Therefore, excess charge at the step edges alters both the CPD voltage and the capacitance, and consequently, the frequency shift. The effect of the step edge charge was observed in both the $\Delta f$ map shown in Fig. 2(d) and the capacitance map shown in Fig. 4. The wider effect on the capacitance map was probably because the capacitance variation map was measured in a different location on the sample surface, where the step could have had a different charge density. Furthermore, the lower number of data points in Fig. 4 than in the $\Delta f$ map of Fig. 2 broadened the profile. Our observation agrees with reports of a variation of electrostatic force across surface steps due to charge transfer to the surface bonds for $\operatorname{Si}(111)-7 \times 7$ and $\operatorname{GaAs}(110)$ surfaces $[13,17,19]$.

Here, we discuss the origin of the fluctuations on the flat terraces shown in Fig. 2. The work function varies on semiconductor surfaces due to charged states at surface steps, charged defects, and impurity atoms. A variation of $\sim 40 \mathrm{mV}$ in the CPD voltage across surface steps was observed for atomically-flat, cleaved $\operatorname{GaAs}(110)$ surfaces by SKPM [19]. A variation of $15-25 \mathrm{mV}$ in the CPD voltage around charged phosphorus and boron atoms was measured by SKPM at $13 \mathrm{~K}$ under carrier depletion at $\mathrm{Si}$ surfaces $[6,7]$. In our measurements, a standard deviation of $30 \mathrm{mV}$ in the $\mathrm{CPD}$ voltage was obtained at room temperature. Moreover, under carrier depletion at the surface, all charges in the depletion region beneath the STM probe contribute to the surface potential. As a result, the individual dopant-related potential is difficult to distinguish in a CPD voltage map at room temperature. However, surface imperfections and charge traps, such as the black spot at $X=140 \mathrm{~nm}$ in Fig. 4, can be recognized [23]. In contrast, the force gradient gives better sensitivity to individual charge location, because the force acting on the probe is dominated by the charge of the dopant atom nearest to the probe.

\section{CONCLUSIONS}

We demonstrated high-resolution measurements of morphology, the force gradient, and the electrostatic potential on oxide-passivated $\mathrm{Si}(111)$ surfaces by an integrated STM-AFM technique. The observed fluctuations in the force gradient maps were attributed to surface imperfections, surface steps, and donor atoms. The results are applicable to monitoring the positions of dopant atoms and charge traps in small Si structures and SOI devices.

\section{Acknowledgments}

This work was supported by NEDO. A part of this work was conducted at the Nano-Processing Facility, supported by IBEC Innovation Platform, AIST, Japan.
[1] S. Roy and A. Asenov, Sceince 309, 388 (2005).

[2] B. E. Kane, Nature 393, 133 (1998).

[3] K. K. Likharev, IEEE Trans. Magn. 13, 242 (1977).

[4] L. Lequn, J. Yu, and J. W. Lyding, IEEE Trans. Nanotechnol. 1, 176 (2002).

[5] M. Nishizawa, L. Bolotov, and T. Kanayama, Appl. Phys. Lett. 90, 122118 (2007).

[6] M. Nishizawa, L. Bolotov, T. Tada, and T. Kanayama, J. Vac. Sci. Technol. B 24, 365 (2006).

[7] M. Nishizawa, L. Bolotov, and T. Kanayama, Jpn. J. Appl. Phys. 44, L1436 (2005).

[8] L. Bolotov, M. Nishizawa, and T. Kanayama, in AIP CP931: Frontiers of Characterization and Metrology for Nanoelectronics 200\%, Ed. D.G. Seiler, et al. (Springer Verlag, New York, 2007), p. 535.
[9] L. Liu, J. Yu, and J. W. Lyding, Appl. Phys. Lett. 78, 386 (2001).

[10] M. Nishizawa, L. Bolotov, and T. Kanayama, J. Phys.: Conf. Series 106, 012002 (2008).

[11] T. Kanayama, M. Nishizawa, and L. Bolotov, ECS Trans. 19, 117 (2009).

[12] S. Kitamura and M. Iwatsuki, Appl. Phys. Lett. 72, 3154 (1998).

[13] Y. Hasegawa, and T. Eguchi, Appl. Surf. Sci. 188, 386 (2002).

[14] M. Tabe, D. Moraru, M. Ligowski, M. Anwar, K. Yokoi, R. Jablonski, and T. Mizuno, Thin Solid Films 518, S38 (2010).

[15] D. Moraru, M. Ligowski, K. Yokoi, T. Mizuno, and M. Tabe, Appl. Phys. Express 2, 071201 (2009). 
[16] M. Ligowski, D. Moraru, M. Anwar, T. Mizuno, R. Jablonski, and M. Tabe, Appl. Phys. Lett. 93, 142101 (2008).

[17] T. Eguchi, Y. Fujikawa, K. Akiyama, T. An, M. Ono, T. Hashimoto, Y. Morikawa, K. Terakura, T. Sakurai, M. G. Lagally, and Y. Hasegawa, Phys. Rev. Lett. 93, 266102 (2004).

[18] A. K. Henning, T. Hochwitz, J. Slinkman, J. Never, S. Hoffman, P. Kaszuba, and C. Daghlian, J. Appl. Phys. 77, 1888 (1994).

[19] H. O. Jacobs, P. Leuchtmann, O. J. Homan, and A. Stemmer, J. Appl. Phys. 84, 1168 (1998).

[20] A. Kikukawa, S. Hosaka, and R. Imura, Appl. Phys. Lett.
66, 3510 (1995).

[21] T. An, T. Eguchi, K. Akiyama, and Y. Hasegawa, Appl. Phys. Lett. 87, 133114 (2005).

[22] M. Weimer, J. Kramar, and J. D. Baldeschwieler, Phys. Rev. B 39, 5572 (1989).

[23] L. Bolotov, T. Tada, M. Iitake, M. Nishizawa, and T. Kanayama, Jpn. J. Appl. Phys. 50 (2011), in press.

[24] F. J. Giessible, Phys. Rev. B 56, 16010 (1997).

[25] L. Bolotov, N. Uchida, and T. Kanayama, J. Phys.: Condens. Matter 15, S3065 (2003).

[26] E. Yablonovitch, D. L. Allara, C. C. Chang, T. Gmitter, and T. B. Bright, Phys. Rev. Lett. 57, 249 (1986). 